7. Lin G, Walton S, Agboola F, et al. Deflazacort, eteplirsen, and golodirsen for Duchenne muscular dystrophy: effectiveness and value. Final evidence report. Institute for Clinical and Economic Review. August 15, 2019. Available at: https://icer-review.org/wp-content/uploads/2018/12/ICER_ DMD-Final-Report_081519.pdf. Accessed March 11, 2020.

8. Griggs RC, Miller JP, Greenberg CR, et al. Efficacy and safety of deflazacort vs prednisone and placebo for Duchenne muscular dystrophy. Neurology. 2016;87(20):2123-31.

9. Karimzadeh P, Ghazavi A. Comparison of deflazacort and prednisone in Duchenne muscular dystrophy. Iran J Child Neurol. 2012;6(1):5-12. Available at: https://www.researchgate.net/publication/277034512_Comparison_of_ Deflazacort_and_Prednisone_in_Duchenne_Muscular_Dystrophy. Accessed March 11, 2020.

10. Bonifati MD, Ruzza G, Bonometto P, et al. A multicenter, double-blind, randomized trial of deflazacort versus prednisone in Duchenne muscular dystrophy. Muscle Nerve. 2000;23(9):1344-47.

11. McDonald CM, Henricson EK, Abresch RT, et al. Long-term effects of glucocorticoids on function, quality of life, and survival in patients with Duchenne muscular dystrophy: a prospective cohort study. Lancet. 2018;391(10119):451-61.
12. Kim S, Campbell KA, Fox DJ, Matthews DJ, Valdez R. Corticosteroid treatments in males with Duchenne muscular dystrophy: treatment duration and time to loss of ambulation. J Child Neurol. 2015;30(10):1275-80.

13. Bello L, Gordish-Dressman H, Morgenroth LP, et al. Prednisone/prednisolone and deflazacort regimens in the CINRG Duchenne Natural History Study. Neurology. 2015;85(12):1048-55.

14. Mendell JR, Rodino-Klapac LR, Sahenk Z, et al. Eteplirsen for the treatment of Duchenne muscular dystrophy. Ann Neurol. 2013;74(5):637-47.

15. Mendell JR, Goemans N, Lowes LP, et al. Longitudinal effect of eteplirsen versus historical control on ambulation in Duchenne muscular dystrophy. Ann Neurol. 2016;79(2):257-71.

16. Khan N, Eliopoulos H, Han L, et al. Eteplirsen treatment attenuates respiratory decline in ambulatory and non-ambulatory patients with Duchenne muscular dystrophy. J Neuromuscul Dis. 2019;6(2):213-25.

17. Mendell J, Goemans N, Rodino-Klapac L, et al. Eteplirsen, a phosphorodiamidate morpholino oligomer for Duchenne muscular dystrophy: longitudinal comparison to external controls on 6-minute walk test and loss of ambulation [abstract]. Neurology. 2017;88 (16 Suppl):S42.004. Available at: https://n.neurology.org/content/88/16_Supplement/S42.004. Accessed March 11, 2020.

\title{
Evaluating a Stone of Hope: ICER's 2019 Review of Treatments for Duchenne Muscular Dystrophy
}

\author{
John Frederick Brandsema, MD
}

\section{COMMENTARY}

(DMD) is a severe X-linked neurodegenerative disorder that is the most common muscular dystrophy of childhood, affecting about 1 in 3,500 male births ${ }^{1}$; at a large pediatric neuromuscular clinic, the diagnosis may be disclosed to multiple families per month. Typically, this conversation occurs with the family of a young boy around 3 or 4 years of age. The discussion of natural history

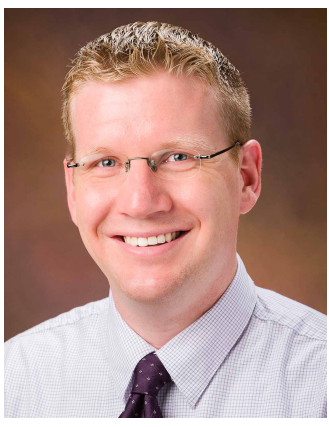

forms of dystrophinopathy, such as the Becker form defined by ambulation beyond age $16 .^{1}$

Before 2016, therapeutic options included optimized interdisciplinary supportive care as well as corticosteroids, the combination of which may increase lifespan into the fourth or rarely fifth decade of life for a larger proportion of patients. Steroids tend to slow but not reverse the relentless decline in muscle strength and are the standard of care in consensus guidelines. The typical effect outlines likely prognostic milestones such as loss of ambulation before age 13, development of detectable and progressive cardiomyopathy and respiratory failure in school age years, and death in the late second or early third decade of life. ${ }^{1}$ Cognition does not decline, but a significant proportion of patients may have cognitive symptoms ranging from behavioral issues such as anxiety or attention deficit hyperactivity disorder to more severe cognitive impairment and autism. There are also milder is around 1-3 years slower disease milestones, but this comes with a host of iatrogenic comorbidities, most prominently increased risk for behavioral issues, linear growth suppression and obesity in younger patients, and eventually poorer bone health with risk for cataracts in older patients. ${ }^{1}$

Treatment options include prednisone/prednisolone versus deflazacort: The ideal steroid type and schedule is still not firmly established since dose reductions due to side effects or family preference, alternative dosing schedules (e.g., daily, weekend-only, and 10 days on and 10 days off), and heterogeneity in the disease phenotype among patients make even matched cohort comparisons have limitations. ${ }^{2}$ 
Access to deflazacort before 2016 was expensive for Americans (approximately \$1,000/year), since it required importation. In February 2017, the U.S. Food and Drug Administration (FDA) label for deflazacort (Emflaza) made insurance coverage a possibility, with even more eligible patients after further expansion of the label from a minimum of 5 to $>2$ years of age in June 2019. This broad label, while more equitable for families that did not have the option of paying previous rates, led to some patients losing their access to deflazacort because of insurance policies-for example, requiring "treatment failure" on prednisone before deflazacort would be approved - and a high burden of authorization for clinical teams. ${ }^{2}$ Given the side effect burden and modest shift of milestones of life-limiting functional loss, patients and their families, as well as provider teams, have long hoped together for more therapeutic options.

The conditional approval of eteplirsen by the FDA in 2016 marked the first Duchennetargeted therapy to receive a label in the United States. An antisense oligonucleotide targeting the skipping of exon 51, approximately $14 \%$ of DMD patients have an amenable mutation in dystrophin that may benefit from this approach. ${ }^{3}$ The approval was based primarily on data from a phase $2 \mathrm{~b}$ randomized controlled clinical trial in 12 boys, 2 of whom lost ambulation soon after enrollment, treated for 24 weeks followed by an open label extension. ${ }^{4}$ Further evidence for efficacy in longer-term follow-up was generated in comparison to a historical cohort in a subsequent publication. ${ }^{5}$ The debate related to this approval hinged on a surrogate marker: slightly increased dystrophin expression in muscle biopsies. Among experts, the quantity and quality of dystrophin expression needed for clinically meaningful benefit remains ambiguous and is continuing to be studied. ${ }^{6-8}$ Tolerability of eteplirsen has shown minimal side effect burden. ${ }^{4}$ Further studies have been ongoing with sparse additional data presented in peerreviewed publications through 2019.

While the initial postapproval excitement was infectious in the Duchenne community, despite the controversy surrounding the approval, it soon became apparent that insurance denials were frequent and that the medication was often not accessible by those who were skip amenable. ${ }^{9}$ Other exon-skipping approaches were in clinical trial at the time of eteplirsen's approval, and it took until December 2019, after a delay due to the FDA's initial rejection of the application in a complete response letter in August 2019, for golodirsen to achieve conditional approval to treat 53-skip amenable patients, an additional $8 \%$ of the Duchenne population, ${ }^{3}$ based primarily again on dystrophin data from muscle biopsies.
Challenges regarding administration of exon-skipping therapies to DMD patients in clinic include the need for a weekly intravenous infusion, requiring central line (port) insertion in patients with challenging peripheral access-common in DMD. In addition, the lack of data in populations earlier or later in their disease course than the population studied in the phase 2 trial led to authorization challenges, as well as complex clinical discussions of benefit versus risk.

On August 15, 2019, the Institute for Clinical and Economic Review (ICER) released its final evidence report on deflazacort, eteplirsen, and golodirsen. ${ }^{10}$ Many of the themes resonate with the Duchenne clinician. Economic modeling attempts to adjust for the variable trajectories and multiple phases of morbidity of DMD (e.g., early and late ambulatory and early and late nonambulatory), but there are some assumptions made in the modeling that oversimplify these phases of decline and their associated comorbidities. Survival is a major focus of the modeling, and there is minimal perspective, likely because of the limited literature for review, on the economic burden and quality of life impact of living with DMD. Such research is somewhat impeded in the United States by historical coding for billing that is not specific to DMD and pools it with other hereditary progressive muscular dystrophies with different spectrums of severities and comorbidities-this improved in the most recent coding classification.

A surprising omission in the clinical benefits section related to steroids is the sparse but nonetheless available literature regarding effect on cardiac function, a major contributor to late-stage morbidity and mortality, and on orthopedic complications such as scoliosis. The bone health aspects of steroid treatment are nuanced, since benefits such as less severe scoliosis are counterbalanced by increased risk for bone fragility and fracture, as is noted in the harms section. When considering high-cost late-stage disease consequences, orthopedic surgeries and cardiac failure admissions are significant contributors, in addition to the respiratory failure exacerbations that can also lead to prolonged hospitalizations for men with DMD.

The ICER report concludes strongly against the pricing of all 3 medications. The voting process yielded a somewhat slim 10-7 majority for the question related to adequate evidence for superiority of deflazacort relative to prednisone. For exon 51 skipping, there was only 1 vote that evidence was adequate to demonstrate a net health benefit when added to the standard of care regimen of corticosteroids plus supportive care. Golodirsen could not be evaluated fully at the time of the report, since there was no available peer-reviewed publications of efficacy and safety data. 
In the clinic, providers and families navigate a complicated decision-making process together in considering these 3 FDAapproved DMD therapies. Questions include the following:

- When is the optimal time to initiate various treatment options (e.g., would newborn screening identification lead to improved outcomes without a tolerability concern regarding early treatment)?

- Are combination therapies more efficacious without decreasing tolerability?

- How does the robustly flowing research pipeline of genetically targeted (e.g., gene transfer, next generation antisense oligonucleotides, and gene editing) and phenotypic modifiers (e.g., antifibrotics and cell-based) affect the use of approved medications?

There is also a challenge of determining when a therapy may no longer be of benefit for those later in their disease course; as the destruction of muscle fibers begins prenatally in DMD and eventually the muscle is replaced by fat and connective tissue, there may be a timepoint when some interventions no longer have the potential to have detectable efficacy. Risk may also be higher with more comorbidities later in the disease. Defining a treatment response or failure is a challenge in an individual patient-while there can be an initial "honeymoon phase" of improvement in function at the initiation of steroids for a few months, the decline phase continues after that point, although with a slightly less negative slope.1 With exon skipping, the concept is one of slower decline; this is challenging to prove in individuals, since their course without the intervention is not known. No matter how well-matched cohorts are based on genotype and phenotype, every person living with DMD has their own experience of the disorder. There are also confounders that can arise, such a fractures with disuse atrophy and a new baseline after recovery that will affect clinical aspects of the disease or biomarkers of severity.

Faced with a daunting challenge of relentless loss of function, individuals living with DMD and their families clamor for therapeutic options to combat their "mountain of despair." Three FDA-approved therapies within approximately 3 years are a "stone of hope" for maintaining function for a few years longer, but there is much work to be done in lessening the burden of this severe neurodegenerative disease. The Duchenne community will continue to strive for further innovation that allows people with dystrophinopathy to "live stronger."

\section{Authors}

JOHN FREDERICK BRANDSEMA, MD, Neurology/Pediatrics, Children's Hospital of Philadelphia, Philadelphia, Pennsylvania.

AUTHOR CORRESPONDENCE: John Frederick Brandsema, MD, Children's Hospital of Philadelphia, Neurology/Pediatrics, Division of Neurology, CTRB 10th Fl., 3501 Civic Center Blvd., Philadelphia, PA 19104.Tel.: 215.590.1719; E-mail: brandsemaj@email.chop.edu.

\section{DISCLOSURES}

No funding contributed to the writing of this commentary. Brandsema reports consulting for Alexion, Audentes, AveXis, Biogen, Cytokinetics, PTC Therapeutics, Sarepta, and WaVe and has received research funding as a site investigator from Alexion, AveXis, Biogen, CSL Behring, Cytokinetics, Fibrogen, Pfizer, PTC Therapeutics, Sarepta, Summit, and WaVe.

\section{REFERENCES}

1. Darras BT, Menache-Stroninki CC, Hinton V, Kunkel LM. Dystrophinopathies. In: Darras BT, Jones HR Jr, Ryan MM, eds. Neuromuscular Disorders of Infancy, Childhood and Adolescence: A Clinician's Approach. 2d ed. San Diego, CA: Academic Press; 2015:551-92.

2. Chrzanowski SM, Poudyal R. Deflazacort-new costs of an old medicine. JAMA Neurol. 2018;75(2):143-44

3. Bladen CL, Salgado D, Monges S, et al. The TREAT-NMD DMD Global Database: analysis of more than 7,000 Duchenne muscular dystrophy mutations. Hum Mutat. 2015;36(4):395-402.

4. Mendell JR, Rodino-Klapac LR, Sahenk Z, et al. Eteplirsen for the treatment of Duchenne muscular dystrophy. Ann Neurol. 2013;74(5):637-47.

5. Mendell JR, Goemans N, Lowes LP, et al. Longitudinal effect of eteplirsen versus historical control on ambulation in Duchenne muscular dystrophy. Ann Neurol. 2016;79(2):257-71.

6. Kesselheim AS, Avorn J. Approving a problematic muscular dystrophy drug: implications for FDA policy. JAMA. 2016;316(22):2357-58.

7. Anthony K, Cirak S, Torelli S, et al. Dystrophin quantification and clinical correlations in Becker muscular dystrophy: implications for clinical trials. Brain. 2011;134(Pt 12):3547-59.

8. Charleston JS, Schnell FJ, Dworzak J, et al. Eteplirsen treatment for Duchenne muscular dystrophy: exon skipping and dystrophin production. Neurology. 2018;90(24):e2146-e2154.

9. Zingariello CD, Kang PB. Dollars and antisense for Duchenne muscular dystrophy: eteplirsen and dystrophin. Neurology. 2018;90(24):1091-92.

10. Institute for Clinical and Economic Review. Deflazacort, eteplirsen, and golodirsen for Duchenne Muscular dystrophy: effectiveness and value. Final evidence report. August 15, 2019. Available at: https://icer-review.org/wpcontent/uploads/2018/12/ICER_DMD-Final-Report_081519-1.pdf. Accessed March 12, 2020 